\title{
BMJ Open Transitioning from paediatric to HIV adult care services for adolescents and young people living with HIV in the African region: a scoping review protocol
}

To cite: Ssemata AS, Nakasujja N, Kinyanda E. Transitioning from paediatric to HIV adult care services for adolescents and young people living with HIV in the African region: a scoping review protocol. BMJ Open 2022;12:e059241. doi:10.1136/ bmjopen-2021-059241

- Prepublication history and additional supplemental material for this paper are available online. To view these files, please visit the journal online (http://dx.doi.org/10.1136/ bmjopen-2021-059241).

Received 16 November 2021 Accepted 04 February 2022

\section{Check for updates}

(c) Author(s) (or their employer(s)) 2022. Re-use permitted under CC BY-NC. No commercial re-use. See rights and permissions. Published by BMJ.

${ }^{1}$ Department of Psychiatry, School of Medicine College of Health Sciences, Makerere University, Kampala, Uganda ${ }^{2}$ Mental Health Section, MRC/ UVRI and LSHTM Uganda Research Unit, Entebbe, Wakiso, Uganda

Correspondence to Andrew Sentoogo Ssemata; andrewssemata@yahoo.co.uk

\section{ABSTRACT}

Introduction The number of children living with HIV is increasing worldwide and is a major public health concern as they grow into adolescence and young adulthood with increasing access to antiretroviral therapy (ART) especially in the African region. There is a pressing need to transfer them from paediatric to adult care which has implications for their well-being. The objective of this scoping review is to systematically review published and unpublished literature to understand the extent and type of evidence in relation to the transition of adolescents to adult HIV clinics in the African region.

Methods and analysis Following the PRISMA-ScR (Preferred Reporting Items for Systematic Reviews and Meta-Analyses-Extension for Scoping Review) guidelines for conducting a scoping review, we will systematically search online bibliographic databases including PubMed, EMBASE, Google Scholar and bibliographies of pertinent articles. This will be supplemented by searches in grey literature databases. Two reviewers will independently review all articles to determine if they meet eligibility criteria. Any conflicts will be resolved after discussion with a third reviewer to ensure accurate and reliable data collection. Both quantitative and qualitative results will be extracted from all included articles and synthesised in a narrative form in response to the review questions. Ethics and dissemination The scoping review does not require ethics approval as we will collect and review existing literature and materials. The results will be disseminated through a peer-reviewed publication, conference presentations and stakeholder meetings to support clinicians, health experts and policy makers develop guidelines and evidence-based transition protocols favourable for the populations in the African region to minimise challenges associated with the transition process.

\section{INTRODUCTION}

At the end of 2020, over 37.7 million (30.245.1 million) people were living with HIV, with approximately 25.4 million in the WHO African region. ${ }^{1}$ Sub-Saharan Africa (SSA) has the highest HIV prevalence rate in the
Strengths and limitations of this study

- This scoping review will systematically identify and explore the challenges, health needs and the implications of adolescents living with HIV in the African region transitioning to adult clinics.

- The scoping review methodology allows us to consider grey literature and a broader range of study designs and methods in answering the review questions and thus obtain a comprehensive overview of the transition from paediatric to adult healthcare for adolescents and young people living with HIV in the African region.

- A comprehensive search strategy has been developed in consultation with a medical/health sciences librarian with a broad operationalisation of transition of care to engender a broad scope of empirical and grey literature sources in order to maximise heterogeneity in the results.

- The review will follow the Preferred Reporting Items for Systematic Reviews and Meta-AnalysesScoping Review guidelines to improve the reporting of the scoping review.

- A limitation is that only articles in English will be reviewed.

world where over $80 \%$ of all HIV infections occurring among adolescents are among girls and young women aged 15-24 years, making them particularly vulnerable and twice as likely to be living with HIV than young men of the same age. ${ }^{2}$

Rapid roll out of antiretroviral treatment programmes has made it possible for perinatal or behavioural HIV-infected adolescents (10-19 years) and young adults (20-24 years) to live through adolescence and adulthood, thereby becoming a growing proportion of the HIV-infected population. ${ }^{1} 3$ Generally adolescents and young people, and especially girls and young women, are one of the most-at-risk groups for HIV in SSA as 
they are at a period of critical life changes. ${ }^{4}$ They are most likely to begin to explore their sexuality, engage in high-risk sexual practices, have early sexual debut and encounters due to peer pressure ${ }^{5-7}$ Their desire to explore and being inquisitive leads them to becoming unfaithful to one sexual partner and therefore have multiple concurrent sexual partners, engage in sex with older partners, engage in transactional and commercial sex for economic and social precariousness, all of which are associated with higher odds of becoming infected with HIV. ${ }^{89}$

Adolescence and young adulthood is one of the most challenging transition periods in a person's life, characterised by identity formation and decisions related to developing a sense of adulthood in the context of fewer social controls. ${ }^{10}{ }^{11}$ Adolescence involves the shift of responsibility for HIV care, from the individual's caregiver to the adolescent themselves, yet in much of Africa, HIV/AIDS treatment, care and support is concentrated on either paediatric or adult care, giving less attention and leaving behind a more vulnerable adolescent population. $^{1213}$

These HIV-positive adolescents and young people (AYP) are at risk of passing on the virus to their sexual partners, potential re-infection by HIV and acquiring sexually transmitted infections due to poor access to adolescent-friendly healthcare services, thereby reducing the value of generalised prevention programmes. ${ }^{24}$ This has implications for adherence to highly active antiretroviral therapy (HAART), resistance to available drugs and retention in care.

Early access to antiretroviral therapy (ART) and support to remain on treatment is therefore critical not only to improve the health of people with HIV but also to prevent further HIV transmission. However, studies have shown that adolescents are more likely than adults or younger children to adhere poorly to their medication and are sometimes difficult to retain in care consistently. ${ }^{12} 14$ These factors are strongly associated with resistance to available antiretroviral therapies. ${ }^{15}$ There is therefore a pressing need to systematically transfer AYP from paediatric to adult care. Importantly, during adolescence, there is a shift in responsibility for HIV care in many respects from caregiver and/or health worker to the adolescent to encourage and support independent self-management of treatment compliance. ${ }^{16}$

Recent systematic reviews and meta-analyses on adolescent transition focus on specific issues, namely, the facilitators and barriers, sustaining optimal adherence and psychosocial well-being. ${ }^{17-19}$ Initiatives have been established in many countries to promote patient-centred care models, transition protocols and adolescent-friendly clinics that can better serve this category of people. ${ }^{142021}$ However, despite a large number of heterogeneous interventions that are specific for populations in Africa compounded by the high HIV burden, ${ }^{15}$ it remains unclear the extent to which these interventions have successfully managed the adolescent transition ensuring minimal consequences of the transitioning process such as retention in care and treatment adherence.

Furthermore, there is currently no single protocol to manage the adolescent transition and enhance purposeful planned movement that can be managed across multiple care settings in the African context, and moreover, there are no published attempts to synthesise and review literature on the adolescent transition in this context. ${ }^{17}$ Only one review has evaluated the transition of adolescents in the Caribbean, Central America and South America, Eastern Europe and Asia and Pacific regions. ${ }^{19}$ While this review brought attention to this critical issue, it did not consider literature in the African region. There is a gap in knowledge regarding adolescents living with HIV in the African region and their transition to adult care clinics despite Africa bearing the biggest HIV/AIDs infection burden. ${ }^{1-3}$ Additionally, the transition to adult care in many resource-constrained African settings is marked by movement from the paediatric ward to general/adult facilities, or into integrated HIV clinics where all people living with HIV are seen by the same staff in the same place with no care-transition preparations and absence of transition plans. ${ }^{17}$

This scoping review will comprehensively review and summarise the current evidence adolescent transition drawing on evidence from any research methodology, providing an overview of previous research reported in the literature. ${ }^{22}$ This may be helpful in informing clinical care and service delivery, as well as underscoring further areas for investigation and developing transition protocols favourable for the African population. This scoping review emerged from the need to identify literature and evidence on the implications of transitioning to adult clinics for a vulnerable population which still require further investigation. Thus, the objective of the scoping review is to identify recent evidence on adolescent transition paediatric to adult HIV care services for adolescents and young people living with HIV in the African region. Furthermore, this scoping review is intended to map and summarise the literature on the impact of the transition on adolescent health outcomes.

We will achieve this objective by addressing the following review questions:

a. What is known from the existing literature about the transition of adolescents from paediatric to adult HIV clinics in the African region?

b. What is the impact of the transition on adolescents' outcomes such as mental health and well-being, adherence to ART and clinic attendance?

c. What is the nature of evidence relevant to the provision of adolescent friendly HIV services during and after transition into HIV adult clinics in Africa?

This scoping review will identify critical gaps and provide guidance for future research. 
Table 1 Eligibility criteria

\begin{tabular}{ll}
\hline Inclusion criteria & Exclusion criteria \\
\hline $\begin{array}{l}\text { Articles with participants } \\
\text { below 18 years }\end{array}$ & $\begin{array}{l}\text { Articles with participants } \\
\text { above 18 years }\end{array}$ \\
$\begin{array}{l}\text { Studies on transition in HIV } \\
\text { care conducted in an African } \\
\text { country }\end{array}$ & $\begin{array}{l}\text { Studies conducted in other } \\
\text { geographical regions }\end{array}$ \\
$\begin{array}{l}\text { Peer-reviewed articles and } \\
\text { protocols }\end{array}$ &
\end{tabular}

Published commentaries,

policy briefs and opinion

pieces

$\begin{array}{ll}\text { Conference proceedings, } & \text { Website information, social } \\ \text { dissertations/theses and } & \text { media content } \\ \text { abstracts published in peer- } & \\ \text { reviewed journals } & \end{array}$

Published any year

English language Non-English language

\section{METHODS AND ANALYSIS}

The scoping review will involve a mapping of previous studies/publications to explore the status of existing literature about the transition to adult HIV clinics to offer a comprehensive overview of the available evidence. The scoping review will identify a range of key themes related to adolescent transition from various studies that are available and identify knowledge gaps. The methods used herein were developed in accordance with the instructions of the Preferred Reporting Items for Systematic Reviews and Meta-Analyses-Extension for Scoping Review (PRISMA-ScR) guidelines, ${ }^{23} 24$ and in accordance with the Joanna Briggs Institute methodology for scoping reviews based on earlier work. ${ }^{25} 26$ The scoping review reporting will be also written in accordance with these guidelines.

\section{Eligibility criteria}

The scoping review will include publications from all years to date with geographical restrictions to publications in the African continent among individuals below 18 years. Studies will be selected according to the following eligibility (inclusion/exclusion) criteria (see table 1).

We will adopt the PCC (Population (or Participants)/ Concept/Context) framework recommended by Joanna Briggs Institute ${ }^{24}$ (see table 2).

\section{Setting}

Only literature/studies undertaken in countries on the African continent will be eligible for inclusion, due to the high burden and receptivity of HIV infection among adolescents and young people in these settings. ${ }^{13}$

\section{Information sources}

We will systematically search MEDLINE (via PubMed), EMBASE, CINAHL and PsycINFO. We will also search the reference lists of included studies to identify other studies. Our final search terms will include the following
Table 2 The review eligibility criteria based on study population, concept, context (PCC) framework

\begin{tabular}{ll}
\hline PCC element & Definition \\
\hline Population & $\begin{array}{l}\text { Children, adolescents, young people below } \\
18 \text { years of age }\end{array}$ \\
Concept & $\begin{array}{l}\text { HIV/AIDS infection } \\
\text { Transition from paediatric to adult HIV } \\
\text { clinics } \\
\text { Outcomes of transition }\end{array}$ \\
Context & $\begin{array}{l}\text { African countries/region } \\
\text { Primary healthcare settings }\end{array}$ \\
\hline
\end{tabular}

text words: HIV; AIDS; children; adolescents; young people transition; clinics (see online supplemental 1). Further articles will be hand-searched and identified from reference lists of eligible papers and grey literature will also be searched for through Google and websites of relevant national and international organisations. These websites will be searched for additional potentially eligible articles, studies, reports and conference abstracts of relevance to the research questions of this review. There will be no restriction on the study design, or year of publication. This review will also consider qualitative studies for inclusion.

\section{Search strategy}

The search strategy will aim to locate both published and unpublished studies. This was developed by a university librarian. We will search MEDLINE (via PubMed), EMBASE, Cumulative Index to Nursing and Allied Health Literature (CINAHL), and Web of Science databases to identify relevant quantitative and qualitative studies and articles on adolescent transition. Any identified additional keywords and potentially useful index terms of these articles were incorporated to develop a full search strategy. The MeSH terms to be used will be selected after searching for each term definition in the MeSH database from PubMed. This scoping review will search journals and other grey literature published outside of journals in order to maximise heterogeneity in the results.

\section{Source of evidence selection and data extraction}

Sources of evidence will include primary research studies, systematic reviews, meta-analyses, letters to the editor, guidelines and searches in other grey literature databases and conference proceedings to allow for the inclusion of all types of evidence. Studies will be included only if they meet the full inclusion criteria above. The abstracts and full-text articles retrieved using the search strategy will be imported into EndNote software (Clarivate Analytics). Duplicate material will be removed and two of the authors (reviewers) will independently screen studies for inclusion against the eligibility criteria at two levels: (1) title and abstract and (2) full text.

The two reviewers will independently assess in detail the full-text articles of studies that meet eligibility to ensure accuracy and extract the data. Reasons for 


\begin{tabular}{ll}
\hline Table 3 Preliminary data extraction/charting table \\
\hline Item & Description \\
\hline Author(s) & \\
Title & \\
Year of publication & \\
Journal & \\
Country/geographical area & Original research \\
Study type & $\begin{array}{l}\text { Systematic review } \\
\text { Commentary } \\
\text { Protocol } \\
\text { Conference/workshop } \\
\text { proceedings }\end{array}$ \\
& Aim of the publication \\
Study objective & $\begin{array}{l}\text { Age } \\
\text { Gender }\end{array}$ \\
Study population & \\
\hline Outcome/theme &
\end{tabular}

exclusion of sources of evidence on reading the full text will be recorded and reported in the scoping review. The extracted data from the two reviewers will be compared for any discrepancies or disagreements, whereupon another author will serve as a third reviewer to create a consensus and any differences will be resolved through discussion to ensure accurate and reliable data collection.

We will use a data extraction form (see table 3) developed by the reviewers using Microsoft Excel to capture details (eg, authors, year of publication, country of origin); aim/purpose; population characteristics (eg, age, gender, sample size), study design (eg, randomised controlled trials, quasi-experimental, before and after studies, prospective and retrospective cohort studies, casecontrol studies, cohort studies, cross-sectional studies), outcomes and key findings relevant and reported to be associated to the review question/s.

This extraction/charting form will be modified and revised as necessary during the process of extracting data. The Excel spreadsheet and Endnote library will be used to manage records and data throughout the scoping review. The results of the search and the study inclusion process will be reported in full in the final scoping review and presented in a PRISMA-ScR flow diagram to show each level of the review process.

\section{Data synthesis and analysis}

We will narratively synthesise the extracted data from all included articles. The evidence established during the review in response to the review questions will be mapped and presented in tabular and graphical form alongside a detailed narrative summarised through thematic content analysis. $^{27}$

\section{ETHICS AND DISSEMINATION}

The scoping review does not require ethics approval as we will collect and review existing literature and materials.
The scoping review will synthesise evidence on the implications of adolescents living with HIV transitioning to adult clinics in the African region as described in the literature. This review will be the first step to formally identify what is known from the existing published scientific literature. Findings from this scoping review may support clinicians, health experts and policy makers develop guidelines and evidence based transition protocols favourable for the African population to minimise challenges associated with the transition process. The results of this scoping review will be disseminated through submission for publication in an international peer-reviewed journal, policy briefs, scientific meetings and conference presentations. We hope this review will serve as a resource for both researchers and practitioners in planning and designing holistic strategies aimed at successfully and effectively transitioning adolescents with HIV in the African context.

\section{Patient and public involvement statement}

This work analyses existing research studies, and therefore, involves no patients or members of the public.

\section{Study status}

Development of the search strategy for the scoping review is complete. The anticipated date for completion of conducting the search is March 2022. The anticipated start date for the analysis is May 2022 and expected completion date is August 2022.

Acknowledgements The authors would like to thank Siegel Lori and Melissa Vetter, Senior University librarians at the Washington University at St Louis Brown School for the support in establishing the comprehensive search strategy.

Contributors ASS led the conceptualisation and design of this work, drafted the protocol manuscript and will lead the conduct of the scoping review. NN and EK revised the protocol for important intellectual content and will provide conceptual and methods guidance throughout the scoping review. All authors have read, provided critical revisions to the manuscript and approved the final version of the submitted protocol.

Funding This work is part of a fellowship supported by the Eunice Kennedy Shriver National Institute of Child Health \& Human Development (NICHD) and Fogarty International Center (FIC) award number D43TW011541. The content is solely the responsibility of the authors and does not necessarily represent the official views of the National Institutes of Health.

Competing interests None declared.

Patient consent for publication Not applicable.

Provenance and peer review Not commissioned; externally peer reviewed.

Supplemental material This content has been supplied by the author(s). It has not been vetted by BMJ Publishing Group Limited (BMJ) and may not have been peer-reviewed. Any opinions or recommendations discussed are solely those of the author(s) and are not endorsed by BMJ. BMJ disclaims all liability and responsibility arising from any reliance placed on the content. Where the content includes any translated material, BMJ does not warrant the accuracy and reliability of the translations (including but not limited to local regulations, clinical guidelines, terminology, drug names and drug dosages), and is not responsible for any error and/or omissions arising from translation and adaptation or otherwise.

Open access This is an open access article distributed in accordance with the Creative Commons Attribution Non Commercial (CC BY-NC 4.0) license, which permits others to distribute, remix, adapt, build upon this work non-commercially, and license their derivative works on different terms, provided the original work is properly cited, appropriate credit is given, any changes made indicated, and the use is non-commercial. See: http://creativecommons.org/licenses/by-nc/4.0/. 
ORCID iD

Andrew Sentoogo Ssemata http://orcid.org/0000-0003-0060-0842

\section{REFERENCES}

1 World Health Organization. Global progress report on HIV, viral hepatitis and sexually transmitted infections, 2021., in accountability for the global health sector strategies 2016-2021: actions for impact. Geneva: World Health Organization, 2021: 180.

2 UNICEF. HIV and AIDS in adolescents. Turning the tide against AIDS will require more concentrated focus on adolescents and young people 2021, 2021. Available: https://data.unicef.org/topic/ adolescents/hiv-aids/

3 UNAIDS. Global HIV statistics fact sheet 2021. Preliminary UNAIDS 2021 epidemiological estimates 2021, 2021. Available: https:// embargo.unaids.org/static/files/uploaded_files/UNAIDS_2021_ FactSheet_en_em.pdf

4 Mathur S, Pilgrim N, Patel SK, et al. Hiv vulnerability among adolescent girls and young women: a multi-country latent class analysis approach. Int J Public Health 2020;65:399-411.

5 Kar SK, Choudhury A, Singh AP. Understanding normal development of adolescent sexuality: a bumpy ride. J Hum Reprod Sci 2015;8:70-4.

6 Ssebunya RN, Matovu JKB, Makumbi FE, et al. Factors associated with prior engagement in high-risk sexual behaviours among adolescents (10-19years) in a pastoralist post-conflict community, Karamoja sub-region, North eastern Uganda. BMC Public Health 2019;19:1027

7 Iseselo MK, Tarimo EAM, Sandstrom E, et al. Sexual behaviours and practices before and after phase I/II HIV vaccine trial: a qualitative study among volunteers in Dar ES Salaam Tanzania. Int J Environ Res Public Health 2020;17:7193.

8 Ranganathan M, MacPhail C, Pettifor A, et al. Young women's perceptions of transactional sex and sexual agency: a qualitative study in the context of rural South Africa. BMC Public Health 2017; $17: 666$

9 Wamoyi J, Heise L, Meiksin R, et al. Is transactional sex exploitative? A social norms perspective, with implications for interventions with adolescent girls and young women in Tanzania. PLoS One 2019;14:e0214366.

10 Acree ME. Transition of care for youth with HIV. Pediatr Ann 2017;46:e198-202.

11 Becht Al, Nelemans SA, Branje SJT, et al. Identity uncertainty and commitment making across adolescence: five-year withinperson associations using daily identity reports. Dev Psychol 2017;53:2103-12.
12 Mutumba M, Musiime V, Mugerwa H, et al. Perceptions of HIV selfmanagement roles and challenges in adolescents, caregivers, and health care providers. J Assoc Nurses AIDS Care 2019;30:415-27.

13 Gitahi N, Camlin C, Mwania V, et al. Psychosocial needs among older perinatally infected adolescents living with HIV and transitioning to adult care in Kenya. PLoS One 2020;15:e0233451.

14 Lee L, Yehia BR, Gaur AH, et al. The impact of Youth-Friendly structures of care on retention among HIV-infected youth. AIDS Patient Care STDS 2016;30:170-7.

15 Toska E, Pantelic M, Meinck F, et al. Sex in the shadow of HIV: a systematic review of prevalence, risk factors, and interventions to reduce sexual risk-taking among HIV-positive adolescents and youth in sub-Saharan Africa. PLoS One 2017;12:e0178106.

16 Lanyon C, Seeley J, Namukwaya S, et al. "Because we all have to grow up": supporting adolescents in Uganda to develop core competencies to transition towards managing their HIV more independently. J Int AIDS Soc 2020;23 Suppl 5:e25552.

17 Dahourou DL, Gautier-Lafaye C, Teasdale CA, et al. Transition from paediatric to adult care of adolescents living with HIV in sub-Saharan Africa: challenges, youth-friendly models, and outcomes. J Int AIDS Soc 2017;20:21528.

18 Dowshen N, D'Angelo L. Health care transition for youth living with HIV/AIDS. Pediatrics 2011;128:762-71.

19 Bailey H, Cruz MLS, Songtaweesin WN, et al. Adolescents with HIV and transition to adult care in the Caribbean, central America and South America, eastern Europe and Asia and Pacific regions. J Int AIDS Soc 2017;20:21475.

20 Tanner AE, Philbin MM, Duval A, et al. "Youth friendly" clinics: considerations for linking and engaging HIV-infected adolescents into care. AIDS Care 2014;26:199-205.

21 Zanoni BC, Sibaya T, Cairns C, et al. Barriers to retention in care are overcome by Adolescent-Friendly services for adolescents living with HIV in South Africa: a qualitative analysis. AIDS Behav 2019;23:957-65.

22 Peters MDJ, Marnie C, Tricco AC, et al. Updated methodological guidance for the conduct of scoping reviews. JBI Evid Synth 2020;18:2119-26.

23 Tricco AC, Lillie E, Zarin W, et al. PRISMA extension for scoping reviews (PRISMA-ScR): checklist and explanation. Ann Intern Med 2018;169:467-73.

24 Peters MDJet al. Scoping Reviews. In: Aromataris E, Munn Z, eds. JBI manual for evidence synthesis. Joanna Briggs Institute, 2020.

25 Arksey H, O'Malley L. Scoping studies: towards a methodological framework. Int J Soc Res Methodol 2005;8): :19-32.

26 Levac D, Colquhoun H, O'Brien KK. Scoping studies: advancing the methodology. Implement Sci 2010;5: :69.

27 Aveyard H. Doing a literature review in health and social care: a practical guide. 4th edn. London, UK: Open University Press, 2018. 